\title{
ECLIPSE OBSERVATIONS OF IP PEG DURING OUTBURST
}

\author{
W. J. WORRAKER ${ }^{1}$, T. NAYLOR ${ }^{2}$, G. POYNER ${ }^{3}$, \\ S. J. ROBINSON ${ }^{4}$ \\ 1. British Astronomical Association, 65 Wantage Road, \\ Didcot, Oxon., OX11 OAE, UK \\ 2. Department of Physics, Keele University, \\ Staffs, ST5 5BG, UK \\ 3. British Astronomical Association, 67 Ellerton Road, \\ Kingstanding, Birmingham, B44 OQE, UK \\ 4. 178 Fearns Avenue, Bradwell, Stoke-on-Trent, Staffs, UK
}

\section{Introduction}

IP Peg is a U Gem-type dwarf nova with a very high orbital inclination such that the secondary star eclipses the white dwarf primary, accretion disc and hot spot in each orbit. An observing project was set up by 'The Astronomer' (TA) magazine and the British Astronomical Association Variable Star Section to make visual and CCD eclipse observations of IP Peg during outbursts in the 1994/95 season.

\section{Observations}

Two outbursts of IP Peg were observed during the 1994/95 season. The first was detected at $V=12.7 \mathrm{mag}$ by Yamada on August 26.717 UT. The time of minimum light was estimated for 11 eclipses, although the extent and quality of coverage varied considerably.

The second outburst was detected by Worraker at $V=14.3 \mathrm{mag}$ on December 05.855 UT. This outburst was less well covered, and times of minimum light were estimated for only four eclipses.

Visual estimates were in general based on the chart and comparison sequence for IP Peg produced by the editor of TA (latest revision GMH 940130). The maximum visual observing rate was about 1 estimate per minute.

Platt's CCD counts were referenced to star D of the TA sequence at magnitude 12.7 (see [2]), which gave better agreement with visual results 
than the TA value of $V=12.2 \mathrm{mag}$; independent checks suggest that the latter is too bright. Platt's Starlight Xpress camera was used unfiltered, which means that his counts include a possibly significant contribution from the near-infrared part of the spectrum. The integration time was $40 \mathrm{~s}$ early in the August outburst, 80 s later when IP Peg was fainter.

The Keele CCD counts were referenced to star B at magnitude $V=11.0$ [2]. The ST-6 camera was in most cases used with a $V$-filter, which gave good agreement with visual results. A $B$-filter was used instead on two occasions late in the August outburst. The integration time was normally $10 \mathrm{~s}$ with a dead time of $5 \mathrm{~s}$.

Samples of the resulting eclipse profiles are shown in Fig. 1. The times above the figures refer to predicted times of minimum light. The upper panel shows the best-covered eclipse of the year, while the lower panel shows the eclipse at the end of the August outburst, which was covered by both CCD observers.

\section{Eclipse modelling}

Since the eclipses are quite shallow, using them to derive the temperature distribution across a thin disc will result in a radial temperature distribution which is much flatter than steady state. Since the eclipse profiles are stable on a relatively long time-scale (typically $1 . .2$ weeks) compared with the viscous time-scale of the disc (probably around $1 \mathrm{~d}$ ), such a non-steady state could not be maintained for the duration of the plateau phase of the outburst. It is more likely that the mass flux through the disc is close to a steady state distribution, so we have assumed this whilst modelling the eclipses using the code described in [3]. The most important feature of this model is that the accretion disc has an 'opening angle', i.e. flares towards its edge (see Fig. 2).

Most of the parameters of the model are either unimportant (e.g. the disc is so bright that the temperature of the secondary is irrelevant), or fixed by previous workers (e.g. the mass ratio and distance). The only parameters we can vary which have a significant effect on the light curve are the mass transfer rate, disc opening angle, and disc radius. The mass transfer rate fixes the out-of-eclipse magnitude, the disc opening angle the eclipse depth, and the disc radius the eclipse width.

Fig. 3 shows a typical model eclipse overlayed on the data. The depth of the eclipse is well matched. The implied semi-opening angle of the disc is $14^{\circ}$ (for a system inclination of $80^{\circ}$ ). The disc radius used for this model is the tidal radius, and as the model eclipse is a little too narrow, this implies that the disc has grown over the tidal radius for some fraction of the outburst. 

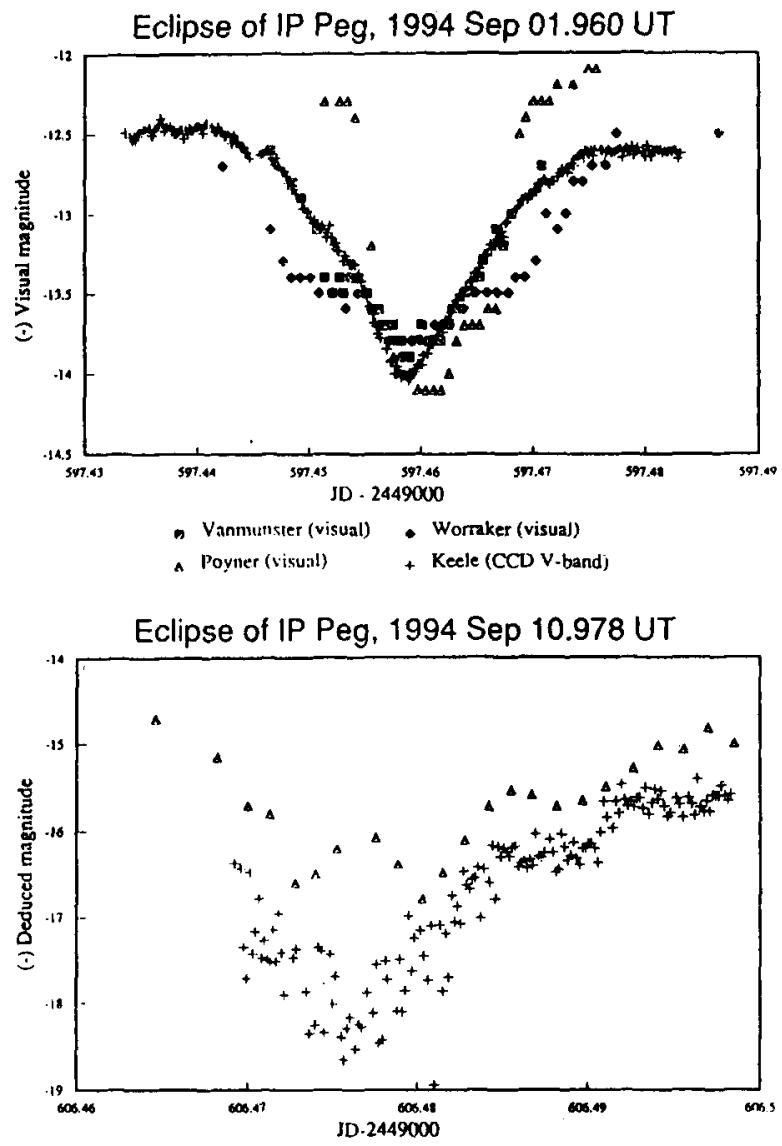

a Platt (CCD unfiliered) + Keele (CCD B-band)

Figure 1. Typical outburst eclipses

Figure 2. The $V$-band light distribution from the model projected onto the plane of the sky. Note that the intensity scale is logarithmic. 


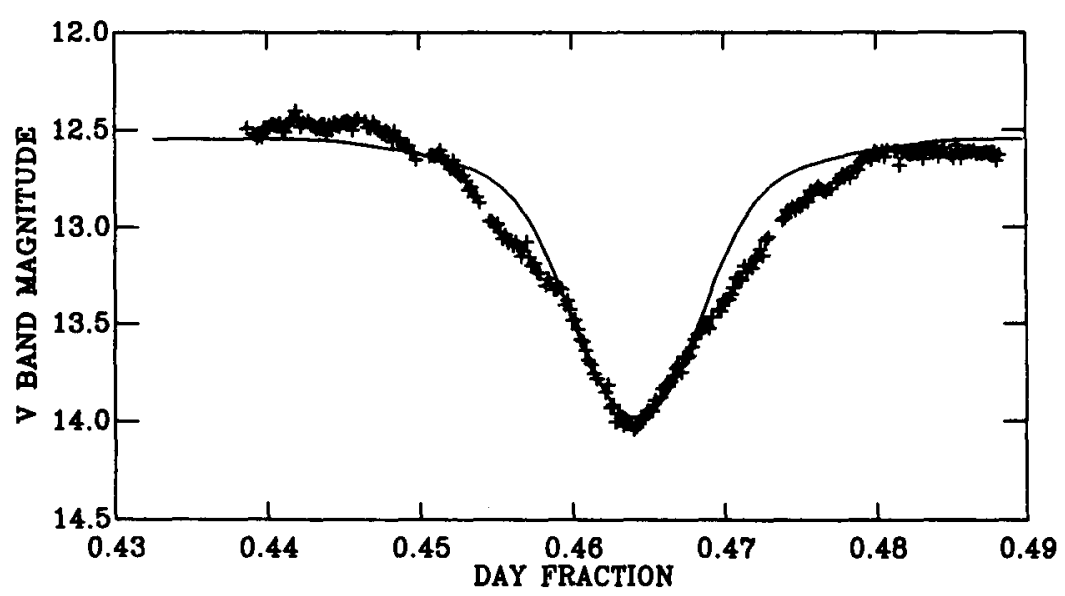

Figure 3. The eclipse resulting from the model shown in Fig. 2 overlayed on a typical eclipse. Note that the real eclipse is wider than the model.

\section{Discussion}

(i) Comparison of observing techniques: The largest errors in the visual estimates are due to the comparison sequence. Thus Poyner's brightest estimates in the upper panel of Fig. 1 are based on star D being at magnitude 12.2 ; the Skiff value of $V=12.7 \mathrm{mag}$ [2] gives better agreement with other results. The CCD results show a number of subtle features on the ingress and egress curves, e.g. changes of slope, which are often missed in the visual results. In some cases this is simply because visual estimates are being made too slowly to give adequate time resolution. On the positive side, the eclipse depth, the time of minimum light and the beginning and end of an eclipse are generally well represented by the visual data.

The plots shown in the lower panel of Fig. 1 combine the results of CCD measurements in different wavebands; Keele were using a $B$-band filter while Platt was using no filter. It seems that the hump observed by Platt around mid-eclipse must have been due to material emitting mainly red and infrared light which would have been filtered out in the Keele system. Whilst interesting, this comparison suggests that filtering of CCD systems is necessary in this kind of work to standardise results; Johnson $V$ - or $B$-band filters are recommended.

(ii) Interpretation of the light curves: Most of the eclipses are broadly similar in duration and are about $1.5 \mathrm{mag}$ deep. However the Ouda station team observed an eclipse 2.6 mag deep on August 26.789 UT, which was very early in the outburst [4]. Combining these results suggests that the main light source in the August outburst was initially very compact but subsequently expanded and stabilised. Later (day 10 onwards) the eclipses 
became deeper again as the outburst light began to fade significantly.

The light curves lack the white dwarf egress feature characteristic of quiescent eclipses, but have well-defined minima allowing timings to within a few seconds. Lack of an up-to-date ephemeris prevents us from interpreting the timings absolutely, but there seems to be a small advance in the time of minimum light relative to the published ephemeris through the August outburst. In three cases there were sufficient observations to show that the light level was about $0.2 \mathrm{mag}$ brighter before the eclipse than after it. We attribute this to the 'orbital hump' effect due to the changing aspect of the hot spot [1]; in quiescence the difference is about $1 \mathrm{mag}$ in the $B$-band $[5,6]$.

Three of the August/September eclipses have been covered sufficiently well (eclipse numbers 25170, 25195 and 25214) for estimates of the total duration of the eclipses to be made. In terms of orbital phase interval (time interval between first and last contact divided by orbital period) these eclipses were $0.214,0.247$ and 0.198 wide respectively. The third value is probably too short as the beginning of the eclipse may have been missed, but the other two suggest a possible increase in the disc diameter between JD 2449597 and 2449601 (days 7 and 11 of the outburst). Definition of the points of first and last contact is intrinsically difficult, so this result should be treated with caution.

Finally we note that the eclipses were all different in detail. There were varying degrees of asymmetry implying time-dependent azimuthal structure in the disc and/or hot spot. However there was no obvious pattern of development of the detailed eclipse structure through an outburst.

\section{Conclusions and recommendations}

(i) Eclipses of IP Peg occurring during its 1994 August/September and 1994 December outbursts have been observed using both visual and CCD techniques.

(ii) The largest source of error in the visual estimates is an inadequate comparison sequence.

(iii) Allowing for problems due to the comparison sequence, the eclipse depth, the time of minimum light and the beginning and end of an eclipse can be determined to a useful degree of accuracy from visual data.

(iv) The visual data represent the details of the ingress and egress light curves to an erratic degree of accuracy.

(v) Unfiltered CCD results may not be comparable with results obtained in standard photometric wavebands.

(vi) In the 1994 August outburst the main outburst light source, which 
must initially have been very compact, spread out and stabilised within about 2 days to give eclipses about $1.5 \mathrm{mag}$ deep in the $V$-band for the plateau phase of the outburst.

(vii) Modelling work suggests that, if we require a steady state disc, to reproduce the eclipse data it is necessary to postulate a flared disc with a semi-opening angle of about $14^{\circ}$.

(viii) In the plateau phase of the 1994 August outburst the orbital hump had an amplitude of about 0.2 mag in the $V$-band.

(ix) The white dwarf egress feature is not visisble in the eclipse light curves obtained during outburst.

(x) Although a recent orbital phase calibration is not available, eclipse minima were approximately $0.002 \mathrm{~d}$ earlier than predicted for most of the 1994 August outburst. However this time difference was smaller earlier (day 5) in the same outburst and on the only occasion (December 13) in the later outburst when it could be measured.

(xi) Eclipses were observed with various degrees of asymmetry and with various changes of slope on the ingress and egress light curves, but there was no discernible pattern of development through an outburst.

In future visual observers should aim to make estimates about once per minute to improve coverage of subtle features of the eclipse light curves. Observations are encouraged for some time both before and after eclipses to check the size of the orbital hump in outburst and its possible relationship with eclipse asymmetries. Accurate white dwarf egress timings made in quiescence are needed to permit full interpretion of minimum light timings made during outburst. More work evidently needs to be done and the IP Peg observing project is continuing.

Acknowledgements. Contributing observers: S. J. Robinson, T. Naylor, T. C. Platt, E. Broens, G. Coady, A. Diepvens, F. Van Loo, G. Poyner, T. Vanmunster, W. Worraker, J. Pietz and M. Westlund.

\section{References}

1. Frank, J., King, A.R., Raine, D.J., 1992, "Accretion Power in Astrophysics" (2nd Edition), Cambridge University Press

2. Skiff, B.A., 1994, VSNET message 'Skiff on IP Peg, issued on 9th October

3. Somers, M. W., Mukai, K., Naylor, T., 1996, MNRAS, in press

4. VSNET postings, August 1994

5. Wood, J. H., Marsh, T. R., Robinson, E. L., et al., 1989, MNRAS, 239, 809

6. Wood, J. H., Crawford, C. S., 1986, MNRAS, 222, 645 